\title{
The Study on Computer Assisted Fresco Painting: A Case Study of the Murals in Xi'an
}

\author{
Yue Ju ${ }^{*}$, Zaosheng Ma \\ Xijing University, Xi’an, 710123, China \\ * Corresponding Author: Yue Ju
}

Keywords: Fresco painting, Murals, Computer assisted painting

\begin{abstract}
Imitating is one of the most important ways for artists to create. Computer aided copying technology is a branch of computer art, which is becoming more and more mature with the development of the times. Taking frescoes in Xi'an as an example, this paper discusses the key technologies, basic process, module construction and application situation of computer aided mural copy to provide some references for relevant researchers.
\end{abstract}

\section{Introduction}

Xi'an is the capital land of Western Han Dynasty and Tang Dynasty, including today's Xi'an city and surrounding counties [1]. In recent years, with the deepening of archaeological work, the relics of the Han and Tang Dynasties in this area have emerged constantly, and the discovery of the Mural Tombs is particularly striking. The discontinuity of the age of the murals in the Han Dynasty in the Han Dynasty in Xi'an was related to the changes in the political, economic and cultural status of the region. The Western Han Dynasty, Xi'an area do, culture is the most prosperous region, but also by the traditional etiquette influence the largest area, especially the influence of social type, funeral ritual upper. As early as the mid-western Han Dynasty, Xi'an area of large and medium-sized tombs are using the traditional ramp ramps soil table burial system. From the West since late coffin burial in the structure is gradually replacing brick structure. The spacious tall brick tombs or tomb, prepare enough space for the tomb murals. The traditional ritual has been a minor role, instead of the life of burial. With the tomb mural decoration is death and ideological development results, when in real life type indoor luxury decoration. There is no lack of fine works in the cultural relics of the Western Han Dynasty unearthed in the Xi'an area. A copper bottles of Xi'an tomb unearthed, inside and outside the body covered with fine patterns, and the cover body wall engraved thin clouds, Ben beast pattern, the cover wall painted clouds, inside painted clouds, dragon, is outside of the bottom painted clouds, the rabbit is a with the typical style of Chu Han Dynasty art. In this paper, we use computer technology such as image processing, artificial intelligence and so on, aiming to study a practical computer aided climbing method that accords with the characteristics of mural restoration [2].

\section{Key Technologies of Computer Assisted Fresco Painting}

\subsection{Smoothing Technology of Line-drawing}

Because of the influence of the disease, the local paint is broken and the wall paintings are broken, and the cracks in the fresco are caused by the aging and temperature change [3]. Therefore, we must first of the mural image smoothing, as far as possible to eliminate noise interference, which is the premise of mural line extraction. This paper uses the norm smoothing, which is a global smoothing filter based on sparse strategy. By sharpening the intensity of transition edge, reducing the smaller intensity jitter to highlight the main structure, we only retain more significant gradient changes. We remove minor non-zero gradients to smooth the unimportant details and enhance the saliency edge of images. Image retouching is the way to process pictures, photos or videos in a way that does not show 
up, including restoring or removing damaged images, or replacing the selected objects in photos. It is also an ancient art creation. As early as the Renaissance, people began to repair some works of art in the middle ages. Their purpose is to fill some cracks to make some medieval paintings renovate. The first step of the computer aided copy of the mural painting is the edge detection and segmentation of the color images. Different from grayscale images, color can also include useful information such as hue and saturation. Color image segmentation can be achieved by computing the statistical characteristics of color information in images. The basic idea of the image algorithm is to propagate the diffusion information smoothly in the direction of the same color line around the area around the area. The user only needs to point out the area to be retraced, and the rest of the work can be completed automatically in a few minutes according to the algorithm. And the painted images are very clear, and there is no artificial color added. This result can be used as a final repair result or provide an initial direction for artificial repair. Therefore, the entire repair time is reduced from the amount of work. A copy of a copy must try to meet the requirements of the original work and cannot be modified [4].

\subsection{Extracting Technology of Line-drawing}

After smoothing, the need for extraction of line drawing pictures. Line extraction is the core of this paper. Generally, the difference of gray value between the pixel and the background pixel in the mural image is larger in the local range, but the range of gray value is overlapped in the global range. To make the pen easier to be extracted, while maintaining the contrast between the pen and the background. First, a high frequency lifting filter is used in smoothing the image in the previous step. Secondly, a new edge tangential flow field is constructed, and then the line map with enhancement effect is extracted. Finally, median filter is used to further eliminate the noise of output image. Based on a new edge tangential flow field, an anisotropic Gauss differential filter is used to extract a smooth line map with an enhanced effect. The filter method is further modified and the features of the murals are strengthened by the high frequency lifting lines. To simulate the stroke style better, we define the template library of stroke samples. We can apply stroke style in template library to new strokes through stroke sample learning algorithm, to achieve better artistic effect. We obtain the feature information of the stroke in the expression model of the stroke library, and then transform the style of a given line. The features of the strokes in the template are matched by the displacement information to the target stroke. All the lines in the target graph are handled above, and the result is the sample style target map and the end of the style conversion process. For the same target graph, many target maps with different style characteristics but consistent content can be obtained by using different control parameters from the above style. By converting style attributes to the target lines, a stroke that has a similar style to the stroke of the sample can be generated.

\subsection{Strengthening Technology of Line-drawing}

In computer aided copying, the simulation of strokes is also a hot issue in the study. Although the computer can't get the creative soul of the artist, it can show the artistry by simulating the characteristics of the stroke. Our simulation of the strokes originates from the trajectory of the contact between the brush and the paper. The simulation of strokes is basically divided into two categories: one is the way of texture mapping, and the other is based on a physical model. From the end of line drawing in the Xi'an murals, is one of the main means of mural techniques and painting procedures. Each time the mural has its different drawing style, has a unique aesthetic value. The line drawing system of Xi'an frescoes can be used not only for the reproduction of auxiliary frescoes, but also for the accurate reproduction of Xi'an frescoes, studying the characteristics of different periods, and on the other hand, it is a special protection method for frescoes. It can be used as the base of mural disease annotation, mural disease monitoring and protection. We give the color space and visual space distance-based image segmentation algorithm, and put forward to line drawing formal expression model for copying works provides a new way of expression and form. We give the piecewise spline curve model related to the drawing and brush strokes, the simple model, the concrete realization method is given. The algorithm is compared with simulation or stroke texture in physical 
model has many advantages based on the calculation, it simplifies the above two methods of complex, texture strokes must be computer simulation based on real time information of strokes, strokes to simulate physical model to generate the stroke information through real-time deformation based on. Based on the stroke simulation of spline curves, simple parameters can be used to express the stroke model information.

\section{Case Study of Computer Assisted Fresco Painting in Xi'an Area}

\subsection{Basic Process}

Image restoration processing is based on the mathematical model of image degradation. To get the image. Not only contains the degradation factors of image itself and there is man-made pollution during image acquisition in the face of many complex factors. If the degradation by establishing model, in the implementation of the rehabilitation of the time efficiency is low. But the comprehensive analysis found that these types of degradation, degradation types can be regarded as a part of the image degradation in linear. Through unified planning ideas. To establish a suitable system simulation model to approximate the degraded image degradation model. We have made a careful study of the facsimile techniques of the murals, and combined with the characteristics of the artists, we put forward the process of computer aided copying. To accomplish this, first through the process of image to large murals, frescoes of image pretreatment, such as contrast and brightness adjustment, image enhancement, image matching algorithm, and then using the edge detection algorithm, such as genetic algorithm, region growing algorithm, clustering algorithm. To get the contour map, the contour map can be vectorization and further annotation. In addition, we use multi-layer rendering technology, the contour extraction of the background image and map background, copying, drawing, break; we combined pressure pen, the curve fitting to each stroke, then the expression of line drawing file. In addition, in order to achieve realistic effect, the wall of the murals must have a certain sense of bump and bump. After the whole wall of the wall was modeled, we began to map the mural parts and the other parts of the wall respectively. For the texture of the other parts of the wall, we can make the texture map of the wall material in advance, and then import the wall to add material. This can more truly reflect the texture of the wall. On the one hand, if the wall is not very smooth in design, we can use the filter to realize the sense of particle in the plane vision, and we can also change the value to change the concave and convex feeling of the wall in the model. This can simulate and greatly enhance the texture of the rough material of the wall. On the other hand, for frescoes, different materials will be used when designing, so when we map, we need to simulate the texture of materials, such as metal and stone. There is a great difference in the degree of refraction, so it is particularly important to adjust the parameters if the later rendering is realized. Line tracing records control parameters of every curve, such as the control point information, pressure information, pen information etc. Since we can also generate line drawing vector amplification. In addition, we can establish the stroke template library, through matching, generated with the artistic style of drawing strokes, finally pressure pen to complete the local details, you can get to the line drawing of the artist. Using the color database by regional growth algorithm to color line drawing, the final shape of the foot in the works.

\subsection{Module Construction}

Preprocessing module: the large mural image can be divided into block processing, since the work efficiency has been improved. The image smoothing is mainly to improve the quality of the mural image, and to remove the noise interference from the crack or the defect of the cell area as much as possible. Image editing is mainly to perform some basic operations on the image, including the functions of image shrinking, rotation, cutting and moving. Layer management module: mainly includes multi-level management, layer establishment, deletion, merging, layer visibility and layer transparency management function, providing a series of auxiliary rendering methods for multi-level rendering. No art skills of users, can help map the mask down painting, if there is good art, you can 
choose to remove the map, according to the original image to depict the free direct control. The mural contour extraction module: including the core function and contour line extraction, vectorization. Line copy module: including pressure pen drawing, line stroke color, selection function width information, revocation, rollback, erase operation function, and the function of the auxiliary according to the choice of the color of color. Line recommendation module: realize the automatic recommendation of line drawing. By drawing vectorization results generated can be recommended to the user, the user through the operation to choose to use part of line drawing recommended, or manually copy themselves. Output module: after the copy is finished, we can save the copy of the result, or print it. If the system is set up in the exhibition area, users can print and take away it immediately after copying, which not only adds pleasure to tourists while waiting, but also allows tourists to have more commemoration. By switching can choose a picture for copying the target, the main area will generate contour and the corresponding map as a copy. The circular toolbar for a floating toolbar, such as map support switch, brush, brush, erase, revocation, rollback, coloring and other functions.

\subsection{Application Situation}

The digital protection research of ancient precious mural paintings and the evaluation of the application and evaluation of the auxiliary system of the copying, constantly improving the key technology and auxiliary system, and ultimately achieving the high-quality demonstration application, and submit all kinds of research summary reports. In the test and application stage, the development of the system is based on the test of the star Xi'an. Different algorithms are implemented for different Xi'an images, and a comprehensive system is developed to handle any quality Xi'an pictures. In Xi'an according to the pictures of different diseases, different colors fade, divided into good quality in three categories, Xi'an Buddha, lotus flower, flying, metaplasia Scout Map, map, and ride the east wall of northern North side musicians Buddha, Buddha and Bodhisattva, Bodhisattva and King figure test, respectively. The contour of the corresponding graph, line drawing, and the vector chart. Record the influence the image quality of the corresponding parameters of the algorithm, structure and optimize the parameters of the input mode, convenient for computer generated line drawings. In the stage of demonstration and application, the developed digital protection and copying system of Xi'an murals has been demonstrated and applied. Computer aided mural copying system provides a new auxiliary copy tools for artists, you can use it to get painting line drawing. It meets the requirements of disease annotation map better, meets the requirements of artists' copying works, and solves the difficult problem of manual duplication. Study on the different historical periods can be the protection of cultural relics experts and artists online tracing based on folk customs and different art style and techniques, summarizes the various historical periods of cultural relics and drawing techniques.

\section{Conclusions}

The application test of computer aided copy system has been applied to the field of computer aided copy of Xi'an murals, and the satisfactory results have been achieved. The research of computer aided murals design has vigorous vitality and has received extensive attention. The exploration of this article opens a new way for the traditional fresco design. Taking the fresco in Xi'an area as an example, it provides a new way for the protection, development and research of Han and Tang culture.

\section{References}

[1] He Zhao, Lu Xuanmin, Wang Junben. Dunhuang Fresco Digital Image Intelligent Mosaics System Based on SURF [J]. Modern Electronics Technique, 2010(16): 133-136.

[2] Jiang Jun, Zhuo Ga, Wang Zhaoxia, et al. Research of Tibet mural digital images inpainting using TV model [J]. Electronic Design Engineering, 2013, 21(3): 136-139. 
[3] Wu Yi, Lu Dongming. Computer Aided Mural Restoration Based on GPU [J].Computer Engineering, 2006, 32(8): 232-234.

[4] Jiang Jun, Wang Longye, Wang Zhaoxia, et al. Research of Tibet mural digital images inpainting using CDD model [J]. Electronic Design Engineering, 2014, 22(2): 177-179. 